\title{
QAGUPUBLICATIONS
}

Journal of Geophysical Research: Planets

Supporting Information for

\section{Properties of Lunar Regolith on the Moon's Farside unveiled by Chang'E-4 Lunar Penetrating Radar}

${ }^{1}$ School of Information and Electronics, Beijing Institute of Technology, Beijing 100081, China.

$8{ }^{2}$ Key Laboratory of Electromagnetic Radiation and Sensing Technology, Aerospace Information Research

9 Institute, Chinese Academy of Sciences, Beijing 100190, China

$10{ }^{3}$ University of Chinese Academy of Science, Beijing 100149, China

$11{ }^{4}$ Greater Bay Area Research Institute of AIR-CAS, Huangpu, Guangzhou, China.

Text S1 to S4

\section{Introduction}

- Text S1: Signal processing methods for LPR data;

- Text S2: Layer localization method for LPR data;

- Text S3: Estimation for dielectric properties of lunar regolith;

- Text S4: Estimation for permittivity of lunar subsurface materials;

- Figure S1: The raw LPR Channel-II data;

- Figure S2: The LPR Channel-II data after DC removing and background removing.;

- Figure S3: The radargram of processed LPR data;

- Figure S4: The frequency spectrum of LPR signal;

- Figure S5: The LPR Channel-II data after Amplitude Compensation; 
29 - Figure S6: The schematic for the layer localization method;

30 - Figure S7: The model of subsurface layers in Li et al. (2020, Sci. Adv.);

31 - Figure S8: Mechanism of the hyperbolic fitting method;

32 - Figure S9: One trace of calibration signals of LPR Channel-II. 


\section{Text S1. Signal processing methods for LPR data}

34 The transmitter of Chang'E-4 (CE-4) lunar penetrating radar (LPR) produces ultra-

35 wide band (UWB) carrier-free nanoseconds pulse and radiates UWB signals downward by

36 transmitting antenna. The signals would reflect and scatter when meeting buried objects

37 during propagation, such as interfaces between different medium, subsurface voids and

38 subsurface boulders. The time window and the sampling point of CE-4 LPR Channel-II

39 are 640 ns and 2048, respectively.

40 Considering the radar system noise and the out-of-band environment noise, the raw

41 LPR data are with low signal-to-noise ratio (SNR) (Fig. S1). Therefore, some processing

42 methods should be performed to improve the SNR of LPR data before further analysis.

43 Here, we employed direct-current (DC) removing, background removing, filtering and

44 amplitude compensation and so on, as usually used for ground penetrating radar data

45 on the earth applications (Jol, 2009; Dong et al., 2016).

46 1. Direct-current (DC) Removing

47 Direct-current (DC) component comes from the oscillation of low frequency during 48 the saturation recovery procedure of receiver. It should be removed before subsequent 49 processing because it doesn't indicate any useful information. The process of DC 50 removing is to slide a window from the beginning to the end of one trace, and then

51 compute the average within the window and subtract the mean value from center value 52 of the window, as shown in equation (S1).

$$
Y(n)=y_{c}-\frac{1}{N} \sum_{1}^{N} y(n)
$$

54 Where, $y_{c}$ and $Y(n)$ represent the values of amplitude before and after processing, 55 respectively. $y(n)$ represents raw LPR data, $N$ is the window length.

\section{2. Background Removing}

57 Background removing aims to eliminate the repetitive and successive interference at 58 the same time delay in every trace. Here we used it to remove the direct wave that 59 coupled from transmitting antenna to receiving antenna, so only the part before surface 60 echoes was processed. The processed amplitude could be derived as equation (S2). 


$$
x^{\prime}(m, n)=x(m, n)-\frac{1}{N} \sum_{n=0}^{N-1} x(m, n)
$$

62 Where $x(m, n)$ is raw LPR data, $x^{\prime}(m, n)$ is processed data, $\mathrm{N}$ is the trace number.

63 The LPR data after DC removing and background removing are shown in Fig. S2.

64 3. Amplitude Compensation

65 The LPR antenna could be approximated to an oscillating dipole that radiates 66 spherical waves. The amplitude of electromagnetic wave that propagates under the

67 ground suffers, therefore, a geometric attenuation due to the distribution of the energy 68 on the front of spherical wave. The amplitude $A(r)$ at the distance $r$ is given as follows:

$$
A(r)=G(r) A_{0} e^{-\alpha r}
$$

70 Where $A_{0}$ is the value of the initial amplitude transmitted from LPR antenna, $\alpha$ is 71 attenuation constant, $G(r)$ is the factor of geometrical spreading which can be derived 72 by equation (S4):

$$
G(r)=\frac{1}{r}
$$

74 Therefore, the amplitude can be compensated by multiplying the LPR signals 75 by $1 / G(r)$. The compensated data show more subsurface information because the 76 deeper signals are amplified, as shown in Fig. S3.

77 4. Filtering Processing

78 A filter in line with the characteristics of LPR data is an effective method to improve 79 the signal-to-noise ratio (SNR). The designed central frequency and bandwidth of CE-4 80 LPR Channel-II are $450 \mathrm{MHz}$ and $500 \mathrm{MHz}$, respectively. Therefore, we chose a band-pass 81 filter with a pass band of 200-700MHz (Fig.S4).

82 From the above methods and procedures, the processed LPR data have improved 83 signal-to-noise ratio compared to the raw data (Fig.S5), which contributes to analyze the 84 subsurface geological structures and estimate the properties of lunar regolith.

\section{Text S2. Layer localization method for LPR data}

86 Because of the high pulse repetition frequency (PRF) of LPR and the low speed of 87 Yutu-2 roving, the LPR echoes of two adjacent traces from the same interface have 
88 similar time delay and amplitude, namely, good correlation. Thus, we can take advantage

89 of the good correlation of two adjacent traces to localize the interface of subsurface

90 layers. However, the buried objects or other underground interference would influence

91 the effect of localization. As a result, we put forward a modified trace correlation method

92 in order to suppress interference and accurately extract subsurface layers, which includes

93 the following steps:

94 (1) Determination of initial values

95 Regard the first trace as reference trace and determine appropriate values for length

96 of reference window and search window, correlation threshold $\left(T_{C}\right)$ and time threshold

$97\left(T_{L}\right)\left(T_{C}\right.$ and $T_{L}$ are empirical values), and then determine initial search point $\left(P_{1}\right)$ by visual 98 inspection.

99 (2) Calculation of correlation

100 Take the next trace as target trace and calculate correlation between the signal in 101 reference window of reference trace and the signal in search window of target trace, and 102 then determine the maximum value of correlation $\left(M_{C}\right)$ and corresponding point $\left(P_{2}\right)$ 103 (Fig.S6).

104 (3) Determination of layer position

105 If $M_{C}>T_{C}$ and $\left|P_{2}-P_{1}\right|<T_{L}$, then consider $P_{2}$ as the layer position of target trace, 106 also as the new search point, regard the target trace as new reference trace. If $M_{C}<T_{C}$, 107 or $M_{C}>T_{C}$ but $\left|P_{2}-P_{1}\right|>T_{L^{\prime}}$ then consider the nearest extreme point from $P_{1}$ as the 108 layer position of target trace, but keep the preceding reference trace and search point.

109 (4) Implement step (2) and (3) to all the traces in order.

\section{Text S3. Estimation for dielectric properties of lunar regolith materials}

111 For LPR data interpretation purposes, the lunar subsurface geological structure at

112 CE-4 landing region could be modeled as a set of three planar layers (i.e., vacuum, 113 surface layer and subsurface layer) (Fig. 3). Each layer is assumed to be semi-infinite that 114 has a magnetic permeability equals to that of free space. 
115 The reflection and transmission coefficients at each layer interface i can be described 116 as follows:

$$
\begin{gathered}
\gamma_{i}=\frac{\sqrt{\varepsilon_{r, i}}-\sqrt{\varepsilon_{r, i+1}}}{\sqrt{\varepsilon_{r, i}}+\sqrt{\varepsilon_{r, i+1}}} \\
\tau_{i}=\frac{2 \sqrt{\varepsilon_{r, i}}}{\sqrt{\varepsilon_{r, i}}+\sqrt{\varepsilon_{r, i+1}}}=1+\gamma_{i}
\end{gathered}
$$

119 The dielectric constant of the surface layer $\left(\varepsilon_{r, 1}\right)$ can be derived from equation (S5):

$$
\varepsilon_{r, 1}=\varepsilon_{r, 0}\left(\frac{1-A_{0}}{1+A_{0}}\right)^{2}
$$

121 Where $\varepsilon_{r, 0}$ is dielectric constant in vacuum. $A_{0}$ is signed relative reflection 122 amplitudes which represents the ratio between the amplitudes of the surface reflection 123 and the amplitude of the incident signal.

124 The relative amplitude of the reflected pulse at the interface between surface and 125 subsurface $A_{1}$ can be given by:

$$
A_{1}=\left(1-\gamma_{0}^{2}\right) \gamma_{1} e^{-\alpha d}
$$

127 where $\alpha$ is attenuation constant, $d$ is thickness of surface layer. Approximate 128 expression for $\alpha$ can be derived as:

$$
\alpha=\sigma_{1} \eta_{0} / 2 \sqrt{\varepsilon_{r, 1}}
$$

130 Where $\eta_{0}=120 \pi$ is wave-impedance in free space. Replacing the reflection 131 coefficient and the attenuation constant with their respective expressions yields the 132 following equation:

$$
A_{1}=\left(1-A_{0}^{2}\right) \frac{\sqrt{\varepsilon_{r, 1}}-\sqrt{\varepsilon_{r, 2}}}{\sqrt{\varepsilon_{r, 1}}+\sqrt{\varepsilon_{r, 2}}} e^{-\eta_{0} \frac{\sigma_{1}}{\sqrt{\varepsilon_{r, 1}}} d_{1}}
$$

134 The conductivity $\sigma_{1}$ can be found by transforming this equation, as given by 135 equation (S11):

$$
\sigma_{1}=\frac{\sqrt{\varepsilon_{r, 1}}}{d_{1}} \frac{1}{\eta_{0}} \log \left[\frac{1-A_{0}^{2}}{-A_{1}} \frac{\sqrt{\varepsilon_{r, 1}}-\sqrt{\varepsilon_{r, 2}}}{\sqrt{\varepsilon_{r, 1}}+\sqrt{\varepsilon_{r, 2}}}\right]
$$

As a result, the loss tangent and $\mathrm{TiO}_{2}+\mathrm{FeO}$ content of lunar regolith can be derived 138 subsequently.

139 Li et al. (2020) found that the bulk density is $\rho_{\text {bulk }}=1.9 \mathrm{~g} / \mathrm{cm}^{3}$ (or $1.67 \mathrm{~g} / \mathrm{cm}^{3}$ 140 depending on their model) for a layer that is $6 \mathrm{~m}$ thick. Hence, Li et al.'s dielectric 141 constant of $\varepsilon^{\prime}=3.5$ corresponds to the average $\varepsilon_{r}^{\prime}$ of the upper 6 -m-thickregolith 
142 material, as shown in Fig. S7. In this study, considering that the permittivity of lunar

143 regolith increases rapidly at shallow depth, but increases more slowly at deeper depths,

144 we assumed the average permittivity $\left(\varepsilon_{r, 1, \text { average }}\right)$ of regolith materials as $\sim 3.6$.

\section{Text S4. Estimation for permittivity of lunar subsurface materials}

146 LPR observes subsurface structures (such as boulders and interfaces) by detecting

147 dielectric discontinuities. As the distance between the antenna and the subsurface object

148 changes with the moving of Yutu-2 rover, single target buried in the subsurface

149 produces parabolic-shaped diffraction (PSD) in the radar cross section.

150 Considering that rocks in the regolith mostly have irregular shapes and the effect of

151 a rock's diameter will be reduced as the depth increases, we approximate the object as a

152 point reflector in order to simplify the model. Fig. S8 demonstrates the mechanism of

153 target detection for LPR. The propagating distance of electromagnetic (EM) wave from

154 LPR to the subsurface object $\left(L_{1}\right)$ and the minimum propagating distance when LPR is

155 over the subsurface object (L) conform to Eq. (S12) based on the geometric relationship:

$$
L_{1}^{2}-\mathrm{L}^{2}=\mathrm{D}^{2}
$$

Where $D$ is the horizontal distance from LPR to the subsurface object, which can be

158 derived according to the location of Yutu-2 rover. Moreover, $L_{1}$ and $L$ could be described 159 as Eq. (S13), respectively:

$$
\mathrm{L}_{1}=\frac{\mathrm{vt}_{1}}{2}, \mathrm{~L}=\frac{\mathrm{vt}}{2}
$$

161 Where $t_{1}$ and $t$ are the two-way propagating time corresponding to $L_{1}$ and $L, v$ is 162 the propagating velocity of EM wave.

163 The velocity of EM wave $(v)$ is determined by the relative dielectric permittivity $\left(\varepsilon_{r}\right)$ 164 as:

$$
v=\frac{c}{\sqrt{\varepsilon_{r}}}
$$

Where $\mathrm{c}$ is the speed of light in vacuum. Replacing the propagating distance in Eq.

167 (S12) and the propagating velocity in Eq. (S13) with their respective expressions yields 168 the following equation:

$$
\varepsilon_{r}=\left(\left(c \sqrt{t_{1}^{2}-t^{2}}\right) / 2 D\right)^{2}
$$


170 As a result, the relative dielectric permittivity of lunar subsurface materials can be 171 calculated with LPR data. 


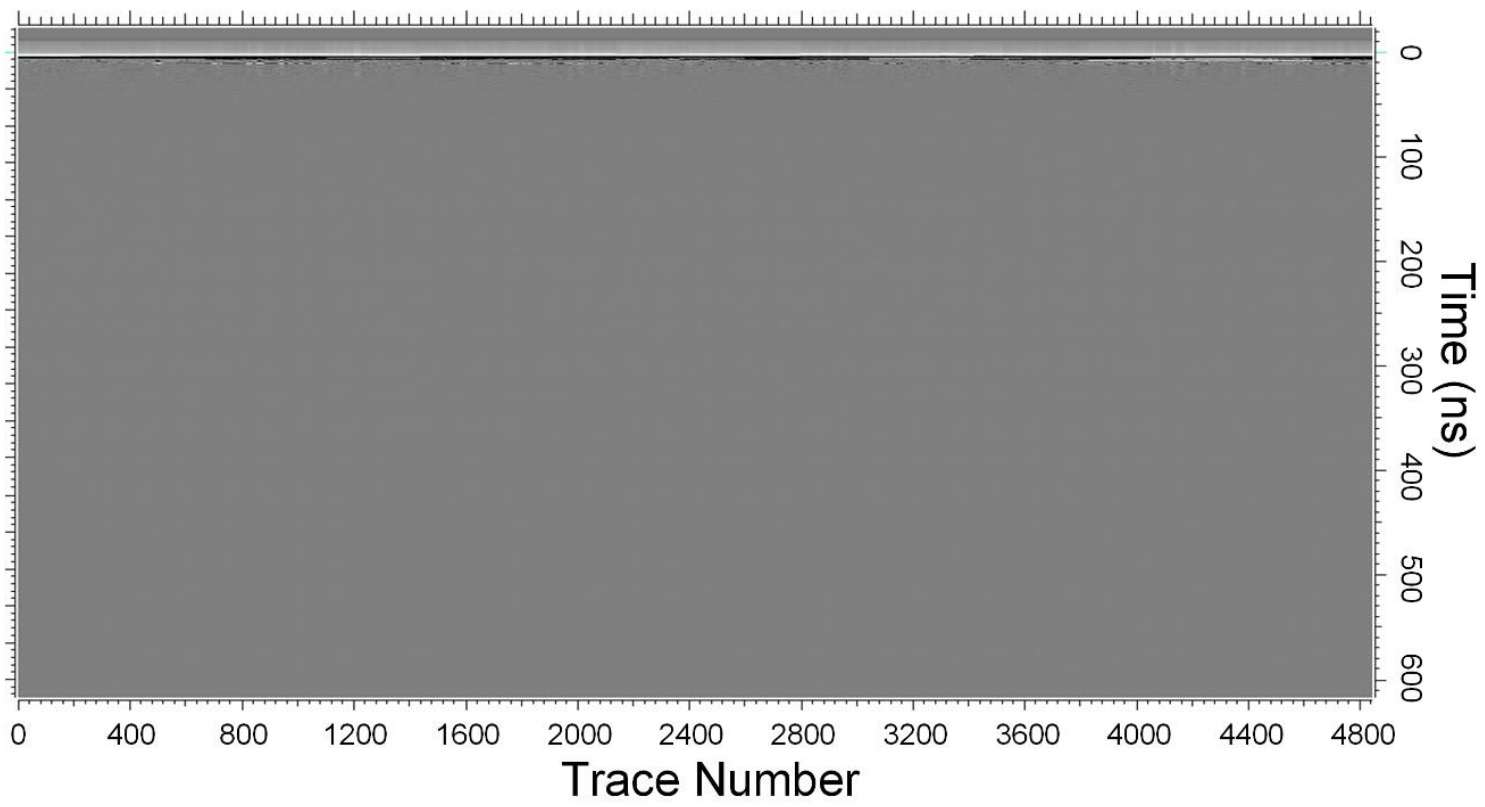

Figure S1. The raw LPR Channel-II data.

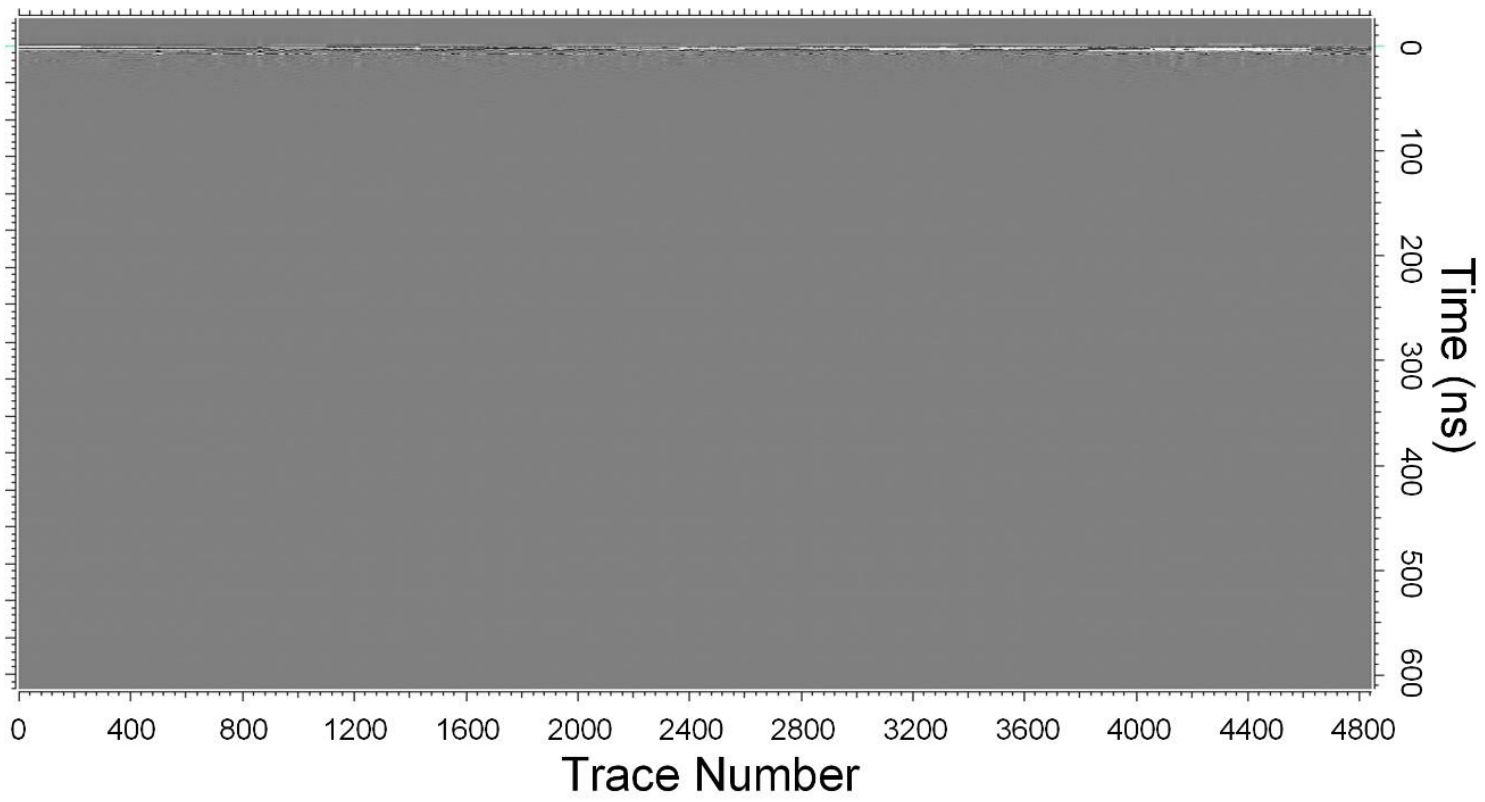

Figure S2. The LPR Channel-II data after DC removing and background removing. 


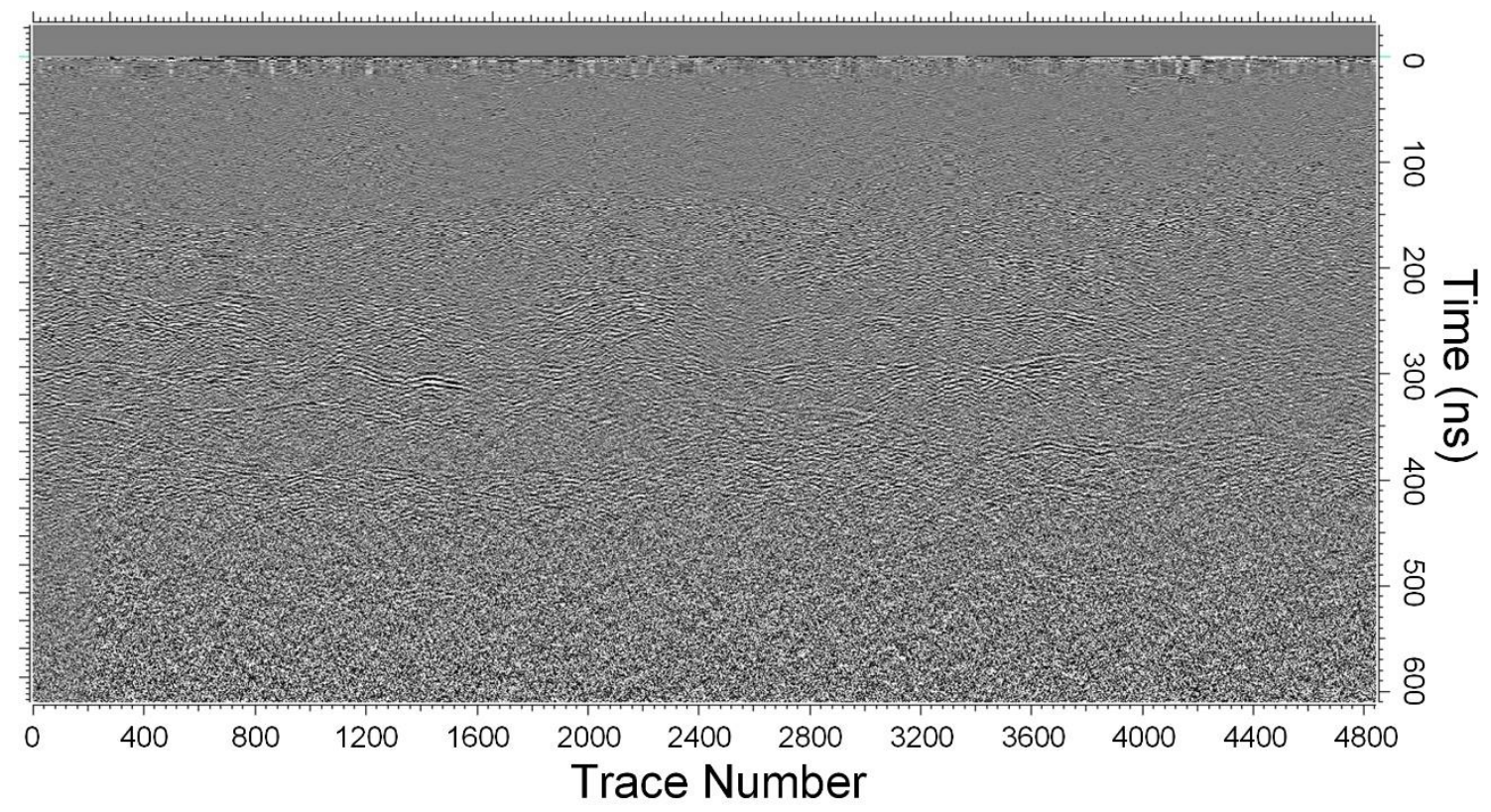

Figure S3. The LPR Channel-II data after Amplitude Compensation.

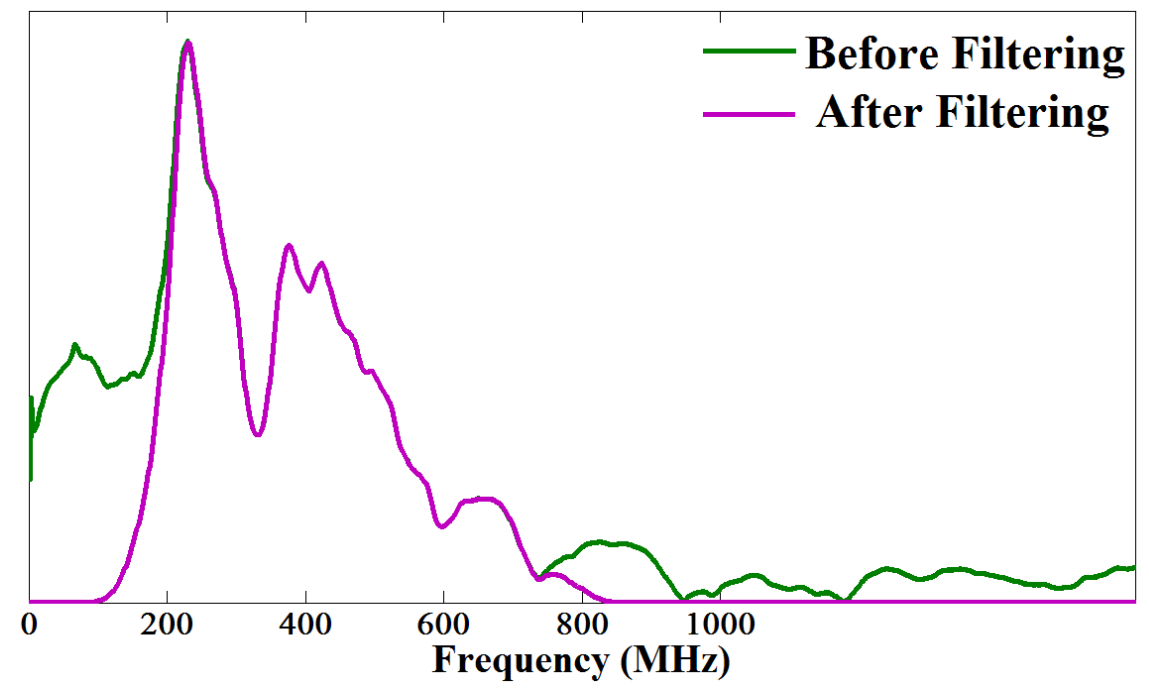

Figure S4. The frequency spectrum of LPR signal before (the green line) and after (the purple line) band-pass filtering. 


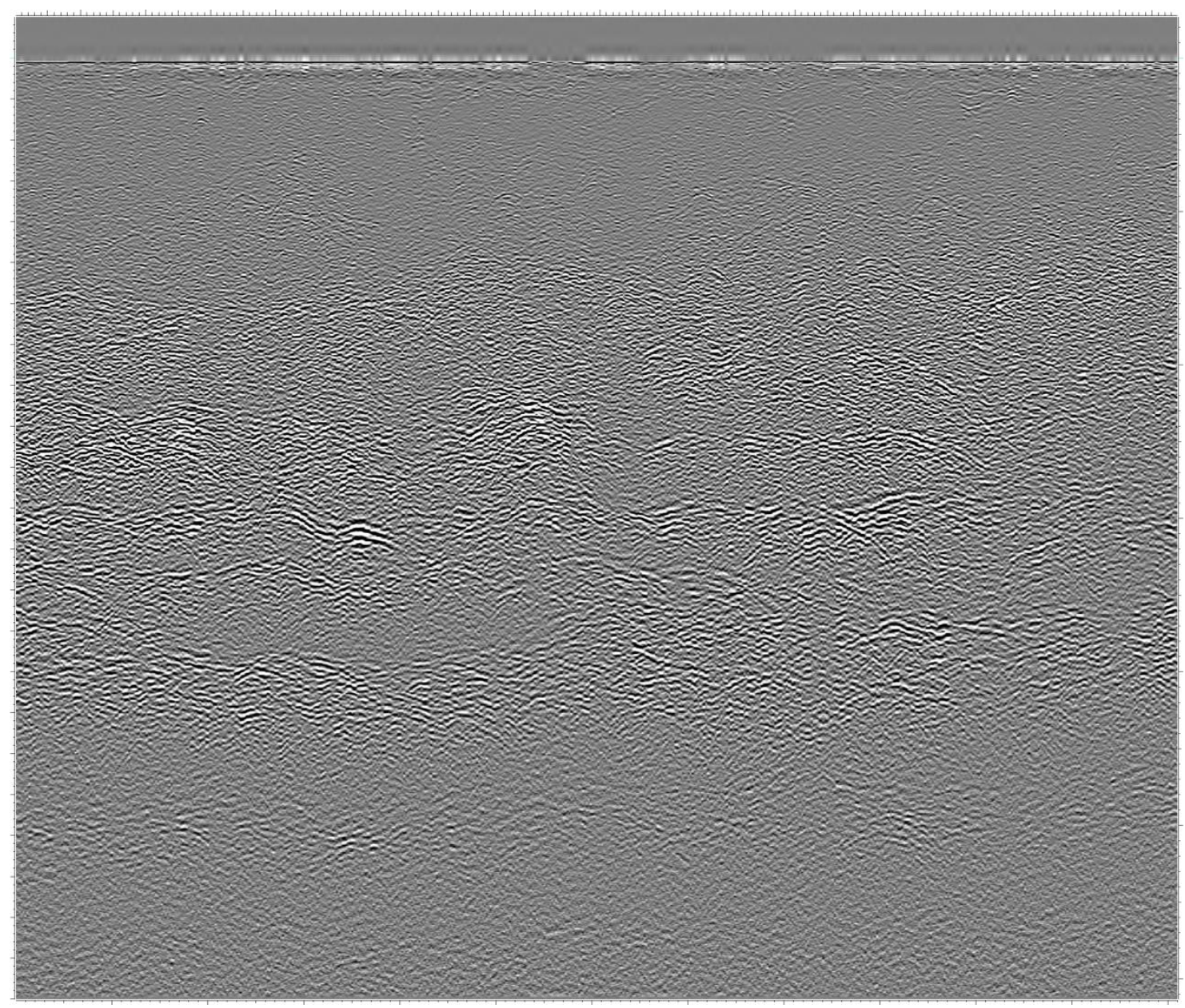

Figure S5. The radargram of processed LPR data after filtering. 


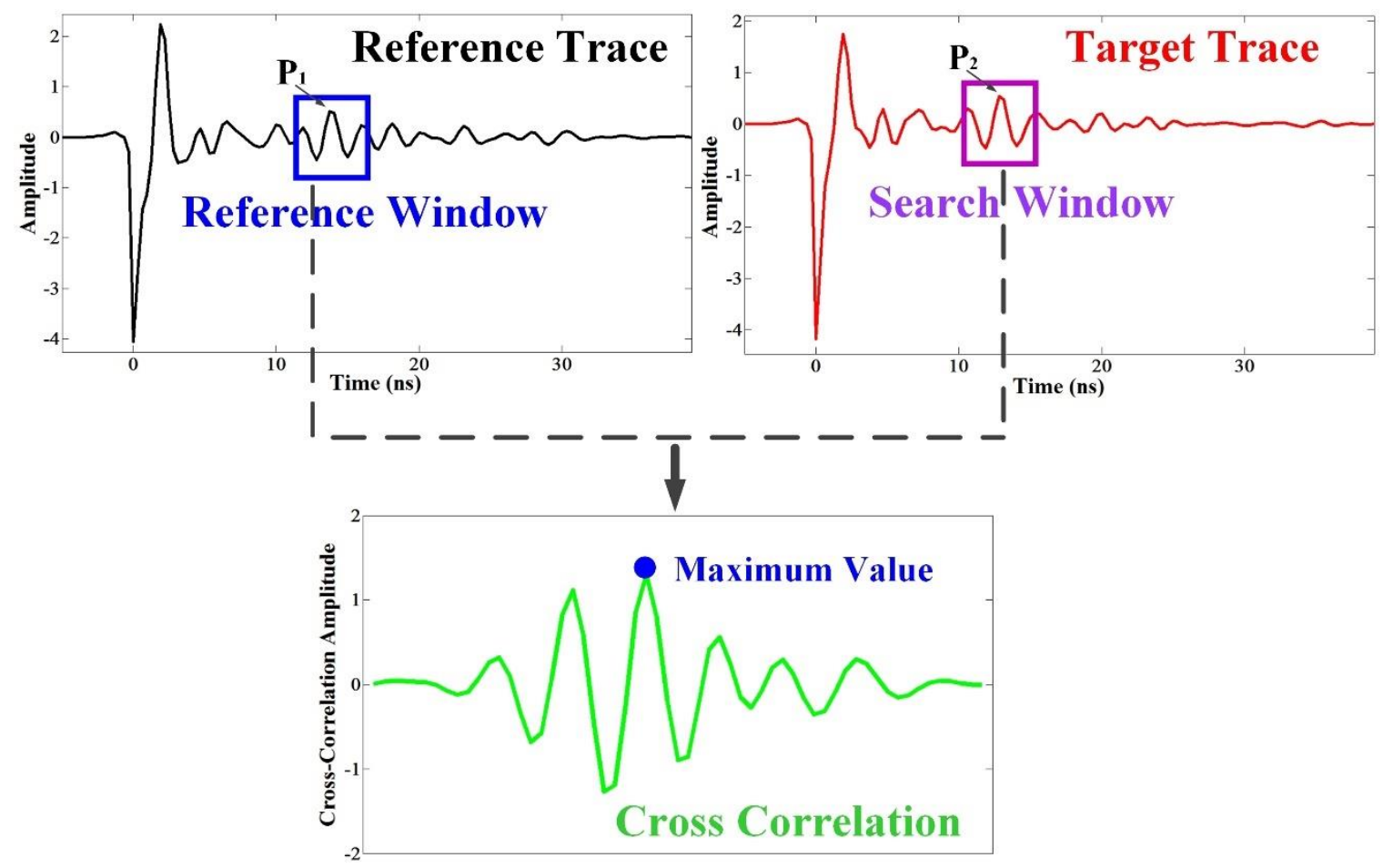

Figure S6. The schematic for the layer localization method. The black signal and red signal indicate reference trace and target trace respectively. The blue and purple rectangles indicate reference window and search window respectively. The search point is the center of both reference window and search window. The green line indicates the cross correlation between signals within reference window and search window. The blue point is maximum value of correlation. 
From Li et al. (2020, Sci. Adv.)
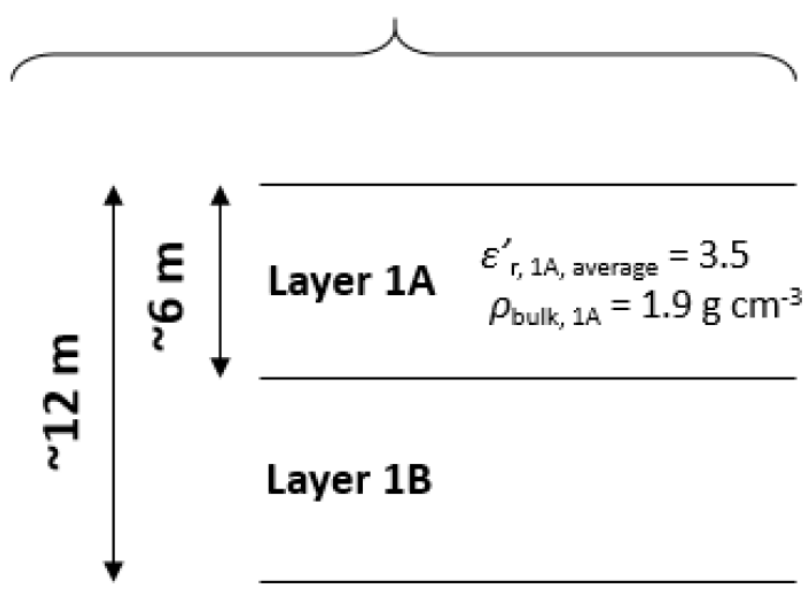

(more layers below)

Figure S7. The model of subsurface layers in Li et al. (2020, Sci. Adv.)

a

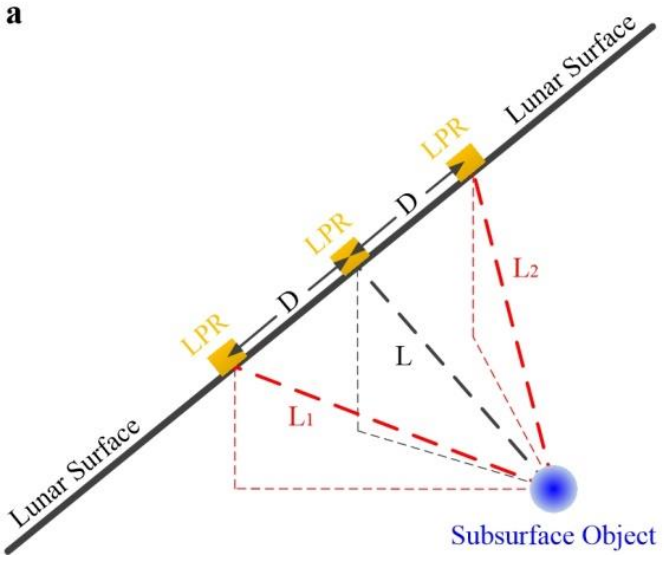

b

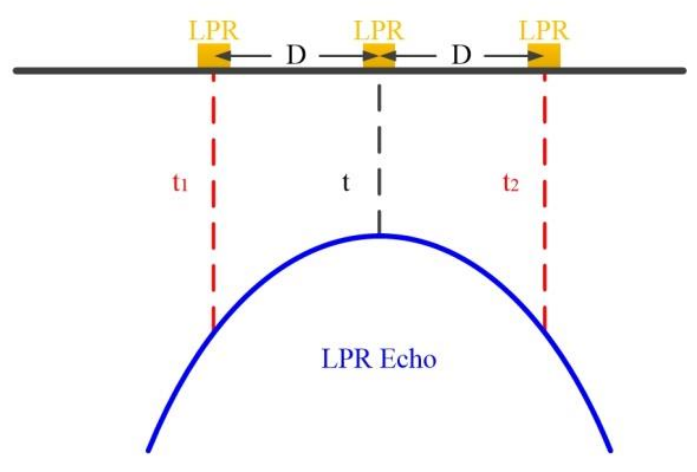

Figure S8. Mechanism of the hyperbolic fitting method. 


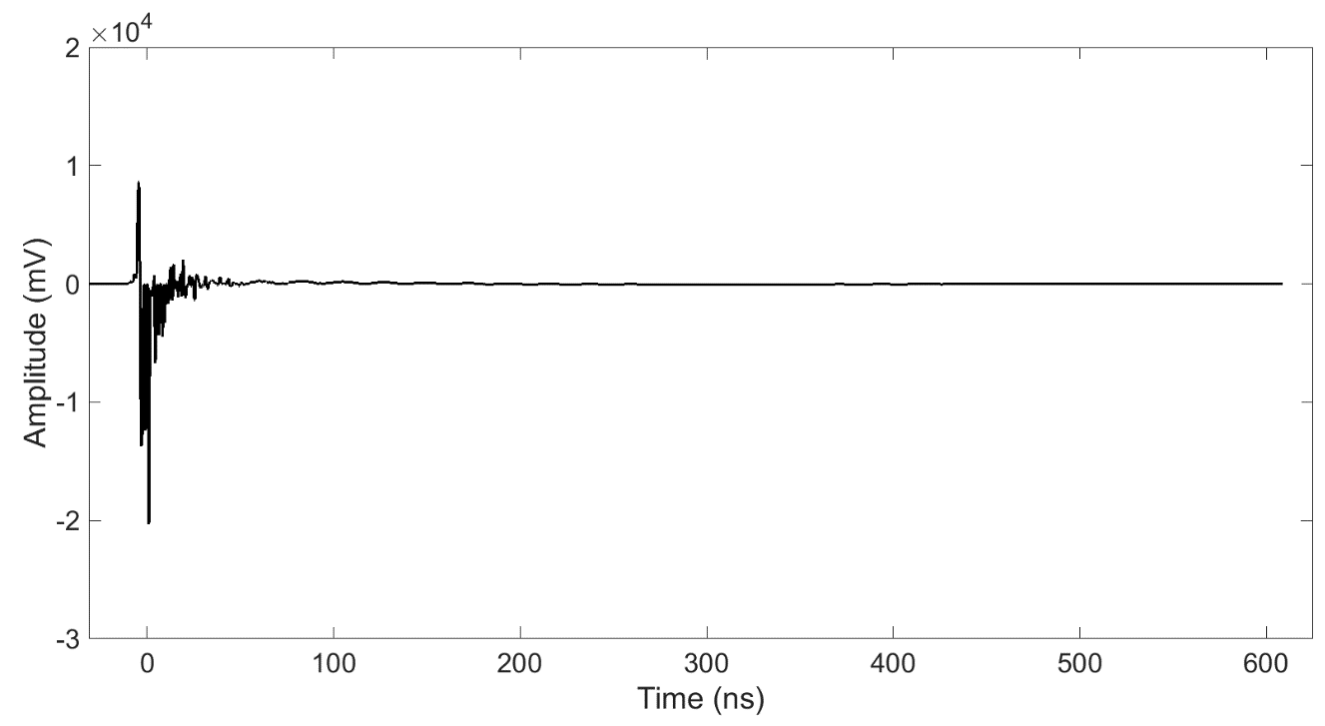

Figure S9. One trace of calibration signals of LPR Channel-II. 